\title{
SATELLITE GUIDANCE AND CONTROL DURING OPERATIVE OPTOELECTRONIC IMAGERY FOR DISASTER MANAGEMENT
}

\author{
Ye. Somov ${ }^{1, *}$, Ch. Hajiyev ${ }^{2}$ \\ ${ }^{1}$ Samara State Technical University, 244 Molodogvardeyskaya Street, Samara 443100 Russia - e_somov@mail.ru \\ ${ }^{2}$ Istanbul Technical University, Maslak, Istanbul 34469 Turkey - cingiz@itu.edu.tr
}

KEY WORDS: Spacecraft, Guidance, Attitude Determination and Control, Areal Land Surveying, Disaster Management

\begin{abstract}
:
We consider problems on surveying the Earth surface during operative optoelectronic imagery for disaster management with respect to attitude guidance and control of the agile spacecraft. The land surveying is carried out by a set of extended orthodromic routes of scanning optoelectronic observation for a given part of the Earth surface. We present developed methods for synthesis of nonlinear guidance and attitude control laws, dynamic research of the spacecraft attitude control system with the satellite astroinertial attitude determination and digital control by the excessive gyro moment cluster. We present results on the efficiency of the developed vector spline guidance laws, algorithms for discrete filtering and the digital gyromoment control of a satellite orientation during the areal landsurveying of Istanbul neighborhoods for the spacecraft on sun-synchronous orbit with altitude of $720 \mathrm{~km}$ when the allowed deviation of the target line from Nadir is within the cone with semi-angle of $40 \mathrm{deg}$.
\end{abstract}

\section{INTRODUCTION}

Dynamic requirements to attitude control system (ACS) for a land-survey spacecraft (SC) are as follows: (i) guidance the telescope's line-of-sight to a predetermined part of the Earth surface with the scan in designated direction; (ii) stabilization of an image motion velocity (IMV) in focal plane (FP) of the onboard optical telescope. These requirements are expressed by the SC rapid angular manoeuvering and spatial compensative motion with a variable angular rate vector, Fig. 1 . Lifetime up to 10 years,

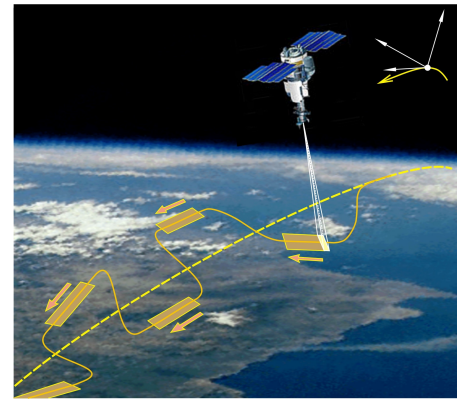

Figure 1. The land-survey SC at imagery of given targets fast spatial rotation maneuvers (RMs) with effective damping the SC structure oscillations, fault tolerance as well as the reasonable mass, size and energy characteristics have motivated development of ACSs equipped with excessive gyro moment clusters (GMCs) based on gyrodines (GDs) - single-gimbal control moment gyros. In the paper we briefly present new results on guidance, precise attitude determination and robust gyromoment attitude control of an agile satellite during operative optoelectronic imagery for disaster management.

\section{MODELS AND THE PROBLEM STATEMENT}

We apply standard bases with the unit vectors and reference frames (RFs) as follows: the inertial RF (IRF) $\mathbf{I}_{\oplus}\left(\mathrm{O}_{\oplus} \mathrm{X}_{\mathrm{I}}^{\mathrm{e}} \mathrm{Y}_{\mathrm{I}}^{\mathrm{e}} \mathrm{Z}_{\mathrm{I}}^{\mathrm{e}}\right)$ with the origin at the Earth center $\mathrm{O}_{\oplus}$; the geodesic Greenwich $\mathrm{RF}$ (GRF) $\mathbf{E}_{\mathrm{e}}\left(\mathrm{O}_{\oplus} \mathrm{X}^{\mathrm{e}} \mathrm{Y}^{\mathrm{e}} \mathrm{Z}^{\mathrm{e}}\right)$ that is rotated with respect to the IRF with the angular rate vector $\boldsymbol{\omega}_{\oplus} \equiv \boldsymbol{\omega}_{\mathrm{e}}$; the horizon $\mathrm{RF}(\mathrm{HRF}) \mathbf{E}_{\mathrm{e}}^{\mathrm{h}}$ $\left(C \mathrm{X}_{c}^{\mathrm{h}} \mathrm{Y}_{c}^{\mathrm{h}} \mathrm{Z}_{c}^{\mathrm{h}}\right)$ with the origin at point $C$ and ellipsoidal geodesic coordinates - altitude $H_{c}$, latitude $B_{c}$ and longitude $L_{c}$; the SC body $\mathrm{RF}(\mathrm{BRF}) \mathbf{B}=\left\{\mathbf{b}_{i}, i=1,2,3 \equiv 1 \div 3\right\}(\mathrm{O} x y z)$ and the

\footnotetext{
${ }^{*}$ Corresponding author
}

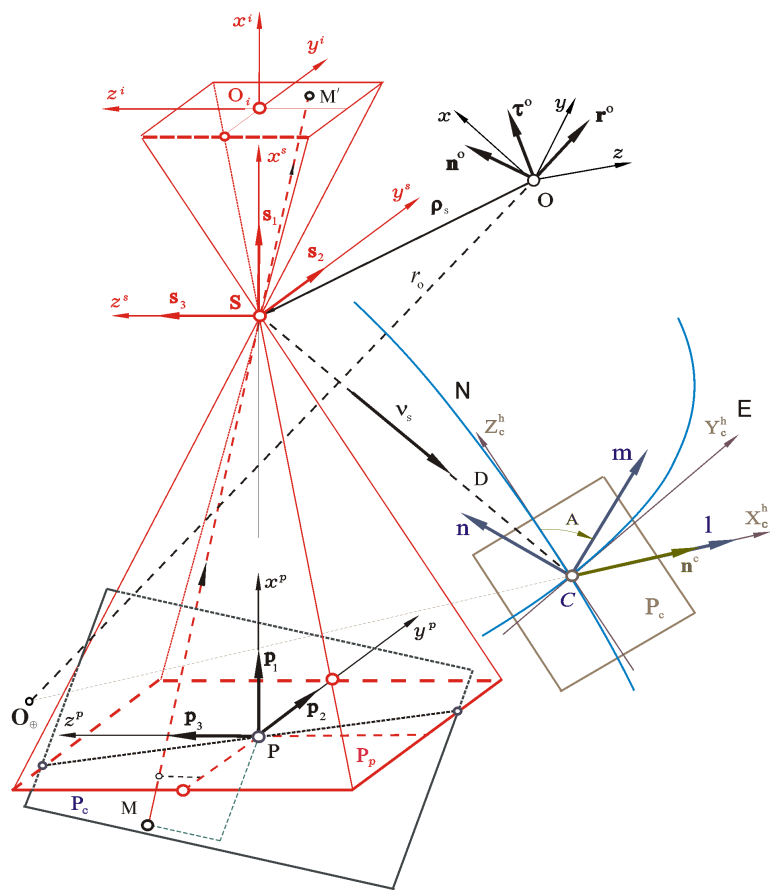

Figure 2. The reference frames for a space land-imagery

orbit RF (ORF) $\mathbf{O}=\left\{\mathbf{r}^{\mathrm{o}}, \boldsymbol{\tau}^{\mathrm{o}}, \mathbf{n}^{\mathrm{o}}\right\}\left(\mathrm{O} x^{\mathrm{o}} y^{\mathrm{o}} z^{\mathrm{o}}\right)$ with the origin in its mass center $\mathrm{O}$; the base $\mathbf{S}=\left\{\mathbf{s}_{1}, \mathbf{s}_{2}, \mathbf{s}_{3}\right\}$ and the sensor reference frame (SRF) of an optical telescope $\mathrm{S} x^{s} y^{s} z^{s}$, Fig. 2; the image field reference frame (FRF) $\mathrm{O}_{i} x^{i} y^{i} z^{i}$ with the origin in center $\mathrm{O}_{i}$ of the telescope focal plane (FP) $y^{i} \mathrm{O}_{i} z^{i}$; the visual RF (VRF) $\mathbf{V}=\left\{\mathbf{v}_{1}, \mathbf{v}_{2}, \mathbf{v}_{3}\right\}\left(\mathrm{O}_{\mathrm{v}} x^{\mathrm{v}} y^{\mathrm{v}} z^{\mathrm{v}}\right)$ with the origin in center $\mathrm{O}_{\mathrm{v}}$ of a CCD matrix in the telescope FP, moreover, points $\mathrm{O}_{i}$ and $\mathrm{O}_{\mathrm{v}}$ are coincident, Fig. 3. The base $\mathrm{A}_{p}=\left\{\mathrm{a}_{p}, \mathrm{~b}_{p}, \mathrm{c}_{p}\right\}$ and the reference frame $\mathrm{STRF}_{p} \mathrm{O} x_{p}^{\mathrm{a}} y_{p}^{\mathrm{a}} z_{p}^{\mathrm{a}}$ of $p$ 's star tracker are connected with the CCD matrix in its focal plane, $p=1 \div 4$, moreover, the $\mathrm{STRF}_{p}$ position is fixed in the BRF, the units $\mathrm{a}_{p}$ of the STs' optical axes belong to the cone's surface with a semi-angle $\gamma^{a}$, Fig. 4, but their actual positions in the BRF are not exactly known. 

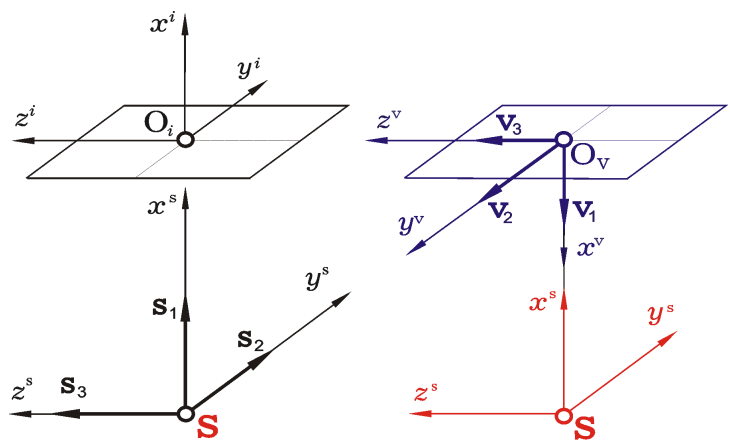

Figure 3. The telescope reference frames

At last, we introduce the virtual base $A=\left\{\mathbf{a}_{1}, \mathbf{a}_{2}, \mathbf{a}_{3}\right\}$ for the star

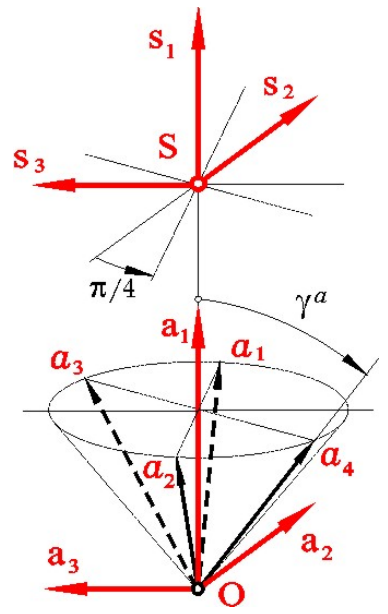
tracker cluster (STC) and its RF $\mathrm{O} x^{\mathrm{a}} y^{\mathrm{a}} z^{\mathrm{a}}$ (STCRF), that is calculated on the basis of proceeding the accessible measuring information from any combination of the STs. For simplicity we will propose that the bases $\mathbf{B}$ and $\mathbf{S}$ (BRF and SRF) coincide. The $\mathrm{BRF}$ orientation in the IRF I $\equiv$ $\mathbf{I}_{\oplus}$ is defined by quaternion $\boldsymbol{\Lambda}_{\mathrm{I}}^{b} \equiv$ $\boldsymbol{\Lambda}=\left(\lambda_{0}, \boldsymbol{\lambda}\right), \boldsymbol{\lambda}=\left\{\lambda_{1}, \lambda_{2}, \lambda_{3}\right\}$ and with respect to the ORF - by column $\phi=\left\{\phi_{i}\right\}, i=1,2,3 \equiv$ $1 \div 3$ of Euler-Krylov angles $\phi_{1}$ (roll), $\phi_{2}$ (yaw) and $\phi_{3}$ (pitch). We use the notations $\boldsymbol{\omega}(t), \mathbf{r}(t)$ and $\mathbf{v}(t)$ for vectors of the SC Figure 4. The bases $\mathbf{S}$ and $\mathbf{A}$ body angular rate, its mass center's position and progressive velocity in the IRF. Here and after symbols $\langle\cdot, \cdot\rangle, \times,\{\cdot\},[\cdot]$ for vectors and $[\mathbf{a} \times],(\cdot)^{\mathrm{t}}$ for matrices are conventional notations.

Collinear pair of two GDs was named as Scissored Pair Ensemble (SPE ) in well-known work J.W. Crenshaw (1973). Minimum redundancy scheme based on 4 gyrodines in the form of 2 collinear pairs, has name 2-SPE. Fig. 5 presents simplest arrangement of this scheme into canonical gyroscopic RF $\mathrm{O} x_{\mathrm{c}}^{\mathrm{g}} y_{\mathrm{c}}^{\mathrm{g}} z_{\mathrm{c}}^{\mathrm{g}}$. By a slope of the GD pair's suspension axes it is possible to change essentially a form of AM variation domain at any direction. The GMC's angular momentum (AM) vector $\mathcal{H}$ has the form $\mathcal{H}(\boldsymbol{\beta})=h_{g} \mathbf{h}(\boldsymbol{\beta})$, there $\mathbf{h}(\boldsymbol{\beta}) \equiv \sum \mathbf{h}_{p}\left(\beta_{p}\right), h_{g}$ is a constant own AM value for GD $\# p=\div 4$ and column $\boldsymbol{\beta}=\left\{\beta_{p}\right\}$. In park state the GMC scheme has the vector of normed $\operatorname{AM} \mathbf{h}(\boldsymbol{\beta})=\mathbf{0}$.

For a fixed position of the SC flexible structures with some simplifying assumptions and $t \in \mathrm{T}_{t_{0}}=\left[t_{0},+\infty\right)$ the $\mathrm{SC}$ angular motion model is appeared as follows

$$
\dot{\boldsymbol{\Lambda}}=\boldsymbol{\Lambda} \circ \boldsymbol{\omega} / 2 ; \mathbf{A}^{o}\{\dot{\boldsymbol{\omega}}, \ddot{\mathbf{q}}\}=\left\{\mathbf{F}^{\omega}, \mathbf{F}^{q}\right\},
$$

where $\boldsymbol{\omega}=\left\{\omega_{i}, i=x, y, z \equiv 1 \div 3\right\} ; \mathbf{q}=\left\{q_{j}, j=1 \div n^{q}\right\}$;

$$
\begin{aligned}
& \mathbf{F}^{\omega}=\mathbf{M}^{g}-\boldsymbol{\omega} \times \mathbf{G}+\mathbf{M}^{d}(t, \boldsymbol{\Lambda}, \boldsymbol{\omega})+\mathbf{Q}^{o}(\boldsymbol{\omega}, \dot{\mathbf{q}}, \mathbf{q}) ; \\
& \mathbf{F}^{q}=\left\{-a_{j}^{q}\left(\left(\delta^{q} / \pi\right) \Omega_{j}^{q} \dot{q}_{j}+\left(\Omega_{j}^{q}\right)^{2} q_{j}\right)+\mathbf{Q}_{j}^{q}\left(\boldsymbol{\omega}, \dot{q}_{j}, q_{j}\right)\right\} ;
\end{aligned}
$$

$\mathbf{A}^{o}=\left[\begin{array}{cc}\mathbf{J} & \mathbf{D}_{q} \\ \mathbf{D}_{q}^{\mathrm{t}} & \mathbf{A}^{q}\end{array}\right] ; \quad \begin{aligned} & \mathbf{G}=\mathbf{G}^{o}+\mathbf{D}_{q} \dot{\mathbf{q}} ; \quad \mathbf{M}^{\mathrm{g}}=-h_{g} \mathbf{A}_{\mathrm{h}}(\boldsymbol{\beta}) \dot{\boldsymbol{\beta}} ; \\ & \mathbf{G}^{o}=\mathbf{J} \boldsymbol{\omega}+\boldsymbol{\mathcal { H }}(\boldsymbol{\beta}) ; \mathbf{A}_{\mathrm{h}}(\boldsymbol{\beta})=\partial \mathbf{h}(\boldsymbol{\beta}) / \partial \boldsymbol{\beta} ;\end{aligned}$

vector $\mathbf{M}^{d}(\cdot)$ presents the external disturbance torques, and $\mathbf{Q}^{o}(\cdot)$, $\mathrm{Q}_{j}^{q}(\cdot)$ are nonlinear continuous functions.

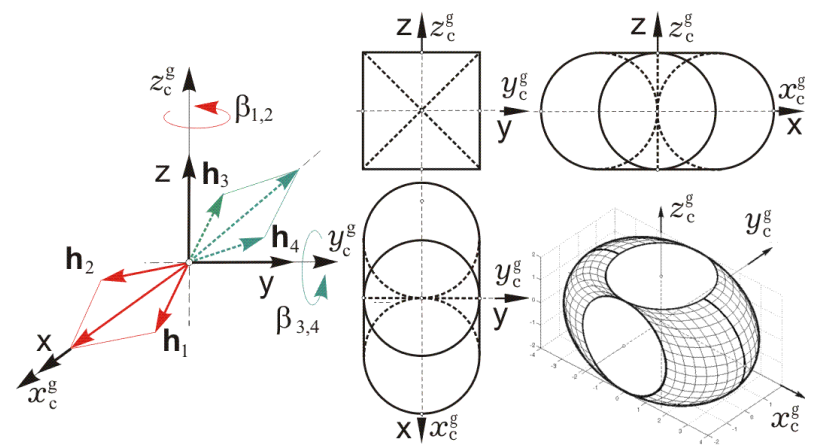

Figure 5. The GMC scheme 2-SPE based on four GDs

The GMC torque vector $\mathbf{M}^{\mathbf{g}}$ is presented as follows

$$
\mathbf{M}^{\mathrm{g}}=\mathbf{M}^{\mathrm{g}}(\boldsymbol{\beta}, \dot{\boldsymbol{\beta}})=-\mathcal{H}^{*}=-h_{g} \mathbf{A}_{\mathrm{h}}(\boldsymbol{\beta}) \mathbf{u}_{k}^{\mathrm{g}} ; \dot{\boldsymbol{\beta}}=\mathbf{u}_{k}^{\mathrm{g}} .
$$

Here $\mathbf{u}_{k}^{\mathrm{g}}=\left\{\mathrm{u}_{p k}^{\mathrm{g}}(t)\right\}, \mathrm{u}_{p k}^{\mathrm{g}}(t)=\operatorname{Zh}\left[\operatorname{Sat}\left(\operatorname{Qntr}\left(u_{p k}^{g}, d^{g}\right), \overline{\mathrm{u}}_{\mathrm{g}}^{\mathrm{m}}\right), T_{u}\right]$ with period $T_{u}=t_{k+1}-t_{k}, k \in \mathbb{N}_{0} \equiv[0,1,2, \ldots)$; functions $u_{p k}^{g} \equiv u_{p}^{g}\left(t_{k}\right)$ are outputs of digital control law, functions $\operatorname{Sat}(x, a)$ and $\operatorname{Qntr}(x, a)$ are general-usage ones, while the holder model has the form $y(t)=\mathrm{Zh}\left[x_{k}, T_{u}\right]=x_{k} \forall t \in$ $\left[t_{k}, t_{k+1}\right)$. At given the SC body angular guidance law $\boldsymbol{\Lambda}^{p}(t)$, $\boldsymbol{\omega}^{p}(t), \boldsymbol{\varepsilon}^{p}(t)=\dot{\boldsymbol{\omega}}^{p}(t)$ during a time interval $t \in \mathrm{T} \equiv\left[t_{\mathrm{i}}, t_{\mathrm{f}}\right] \subset$ $\mathrm{T}_{t_{0}}, t_{\mathrm{f}} \equiv t_{\mathrm{i}}+T$, and for forming the vector of GMC control torque $\mathbf{M}^{\mathrm{g}}(\boldsymbol{\beta}(t), \dot{\boldsymbol{\beta}}(t))(2)$, the vector columns $\dot{\boldsymbol{\beta}}=\left\{\dot{\beta}_{p}\right\}$ and $\ddot{\boldsymbol{\beta}}=\left\{\ddot{\beta}_{p}\right\}$ are component-wise module restricted

$$
\left|\dot{\beta}_{p}(t)\right| \leq \overline{\mathrm{u}}_{\mathrm{g}}<\overline{\mathrm{u}}_{\mathrm{g}}^{\mathrm{m}}, \quad\left|\ddot{\beta}_{p}(t)\right| \leq \overline{\mathrm{v}}_{\mathrm{g}}, \forall t \in \mathrm{T}, p=1 \div 4,
$$

where values $\overline{\mathrm{u}}_{\mathrm{g}}$ and $\overline{\mathrm{v}}_{\mathrm{g}}$ are some positive constants.

At simplest modeling of the SC body with a fixed telescope as a free solid, its AM vector is $\mathbf{G}^{o}=\mathbf{G}_{0}^{o} \equiv \mathbf{0}$ when the satellite ACS is balanced on the AM. Moreover, the model of the SC attitude dynamics has the form $\dot{\omega}=\varepsilon$, where $\varepsilon=\mathbf{J}^{-1} \mathbf{M}^{\mathrm{g}}$ is vector of angular acceleration, and the model of SC attitude motion has the following kinematic representation

$$
\dot{\boldsymbol{\Lambda}}(t)=\boldsymbol{\Lambda}(t) \circ \boldsymbol{\omega}(t) / 2 ; \dot{\boldsymbol{\omega}}(t)=\boldsymbol{\varepsilon}(t) ; \quad \dot{\boldsymbol{\varepsilon}} \equiv \boldsymbol{\varepsilon}^{*}(t)=\mathbf{v} .
$$

Modules of vectors $\boldsymbol{\omega}(t), \boldsymbol{\varepsilon}(t)$ and $\boldsymbol{\varepsilon}^{*}(t)$ are restricted, namely $|\boldsymbol{\omega}(t)| \leq \bar{\omega},|\varepsilon(t)| \leq \bar{\varepsilon}$ and $\left|\varepsilon^{*}(t)\right| \leq \bar{\varepsilon}^{*}$, that is connected with a limited envelop of the variation domains for the GMC vectors of the $\mathrm{AM} \mathcal{H}$ and control torque $\mathbf{M}^{\mathrm{g}}$ with permissible rate of its variation. We apply the modified Rodrigues parameters (MRP) vector $\boldsymbol{\sigma}=\left\{\sigma_{i}\right\}=\mathbf{e} \operatorname{tg}(\Phi / 4)$ with Euler unit vector $\mathbf{e}$ and angle $\Phi$ of own rotation. Vector $\sigma$ is one-one connected with quaternion $\boldsymbol{\Lambda}$ by straight $\boldsymbol{\sigma}=\boldsymbol{\lambda} /\left(1+\lambda_{0}\right)(\boldsymbol{\Lambda} \Rightarrow \boldsymbol{\sigma})$ and reverse $\lambda_{0}=\left(1-\sigma^{2}\right) /\left(1+\sigma^{2}\right) ; \boldsymbol{\lambda}=2 \boldsymbol{\sigma} /\left(1+\sigma^{2}\right)(\boldsymbol{\sigma} \Rightarrow \boldsymbol{\Lambda})$ relations. For vector $\sigma$ kinematic equations have the form

$$
\begin{aligned}
& \dot{\boldsymbol{\sigma}}=\mathbf{F}^{\sigma}(\boldsymbol{\sigma}, \boldsymbol{\omega}) \equiv \frac{1}{4}\left(1-\sigma^{2}\right) \boldsymbol{\omega}+\frac{1}{2} \boldsymbol{\sigma} \times \boldsymbol{\omega}+\frac{1}{2} \boldsymbol{\sigma}\langle\boldsymbol{\sigma}, \boldsymbol{\omega}\rangle ; \\
& \boldsymbol{\omega}=4\left[\left(1-\sigma^{2}\right) \dot{\boldsymbol{\sigma}}-2(\boldsymbol{\sigma} \times \boldsymbol{\sigma})+2 \boldsymbol{\sigma}\langle\dot{\boldsymbol{\sigma}}, \boldsymbol{\sigma}\rangle\right] /\left(1+\sigma^{2}\right)^{2},
\end{aligned}
$$

its second derivative is presented as follows

$$
\begin{aligned}
\ddot{\boldsymbol{\sigma}}= & \frac{1}{2}\left[-\langle\boldsymbol{\sigma}, \dot{\boldsymbol{\sigma}}\rangle \boldsymbol{\omega}+\frac{1}{2}\left(1-\sigma^{2}\right) \boldsymbol{\varepsilon}+\dot{\boldsymbol{\sigma}} \times \boldsymbol{\omega}+\boldsymbol{\sigma} \times \boldsymbol{\varepsilon}\right. \\
& +\dot{\boldsymbol{\sigma}}\langle\boldsymbol{\sigma}, \boldsymbol{\omega}\rangle+\boldsymbol{\sigma}\langle\dot{\boldsymbol{\sigma}}, \boldsymbol{\omega}\rangle+\boldsymbol{\sigma}\langle\boldsymbol{\sigma}, \boldsymbol{\varepsilon}\rangle] .
\end{aligned}
$$

We have applied the attitude determination system (ADS) which contains an inertial measuring unit (IMU) and astronomical system (AS) based on the STC with the star trackers fixed in the SC body. The ADS is a part of the strapdown inertial navigation system (SINS) which solves the general navigation problem - determine both orientation and location of a satellite. 


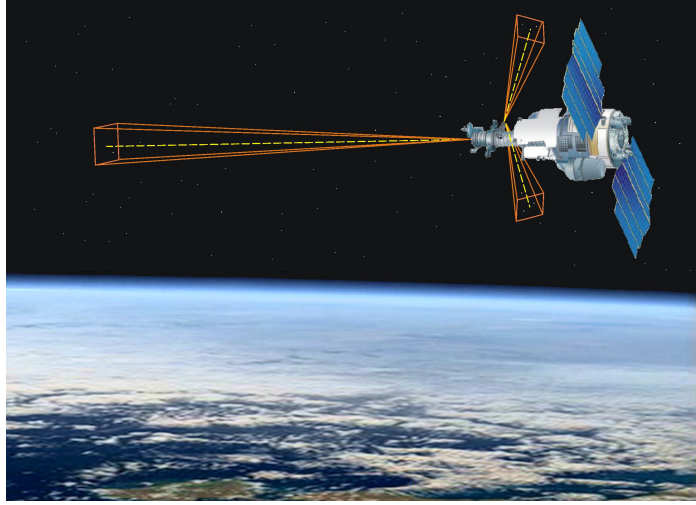

Figure 6. The astronomical checking axes' concordance

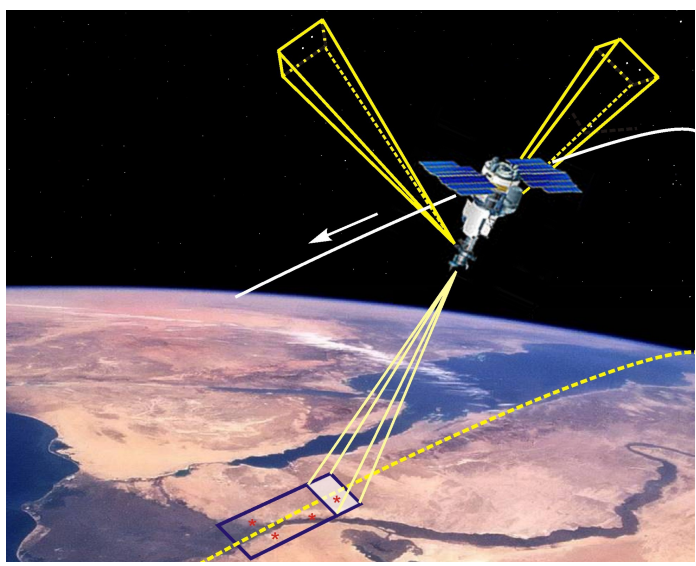

Figure 7. Alignment scheme of a telescope and the STC

A special mode may be organized for mutual binding the SRF and STCRF, when a telescope scans the star sky and simultaneously the optoelectronic STs' measurements are registered, Fig. 6. For the alignment verification other mode is based on observing the terrestrial marked objects by the telescope, Fig. 7. Many customers have own software for processing electronic images and they order only the preprocessed video-information on strictly specified terrestrial parts but together with a service information on the actual conditions of space imagery. Here priority challenge is to develop methods for a more accurate definition of actual mutual position of the telescope and AS by a processing of the measurement information directly aboard a spacecraft.

The problems of the ADS signal processing are connected with integration of kinematic equations in using the information only on the quasi-coordinate increment vector obtained by the IMU at availability of noises, calibration (identification of the IMU bias $\mathbf{b}^{\mathrm{g}}$ and variation $m$ of the measure scale factor) and alignment (identification of errors on a mutual position of the IMU G and AS A reference bases) by the AS signals with the main period $T_{o}$. Many authors applied quaternion $\boldsymbol{\Lambda}$, an orientation matrix $\mathbf{C}$, Euler vector $\boldsymbol{\phi}=\mathbf{e} \Phi$, terminal rotation vector $\boldsymbol{\rho}=2 \mathbf{e} \operatorname{tg}(\Phi / 2)$ etc. Moreover, for the SC low angular motion with a small variation of angle $\Phi$ during period $T_{o}$ and almost fixed Euler unit $\mathbf{e}$, integrating kinematic relation for Euler vector $\phi(t)$ with calculation of values $\Lambda_{r} \equiv \Lambda\left(t_{r}\right)$ is carried out by the scheme

$$
\begin{gathered}
\delta \phi_{r}=\mathbf{i}_{r}^{\omega}=\int_{t_{r}}^{t_{r+1}} \boldsymbol{\omega}(\tau) d \tau \equiv \operatorname{Int}\left(t_{r}, T_{o}, \boldsymbol{\omega}(t)\right) ; \\
\boldsymbol{\phi}_{r}+\delta \boldsymbol{\phi}_{r}=\boldsymbol{\phi}_{r+1} \Rightarrow \mathbf{C}_{r+1} \Rightarrow \boldsymbol{\Lambda}_{r+1},
\end{gathered}
$$

where $\delta \phi_{r}=\delta \Phi_{r} \mathbf{e}_{r}, t_{r+1}=t_{r}+T_{o}, r \in \mathbb{N}_{0}$. Angular movements of a maneuvering land-survey SC are performed on sequence of the time intervals for the observation scanning routes (SRs) and quick rotational maneuvers (RMs) with variable direction of angular rate vector $\boldsymbol{\omega}$ when its module $\omega$ up to $\omega^{\mathrm{m}}=3 \mathrm{deg} / \mathrm{s}$. Assume that the measured values of the quasi-coordinate increment vector $\mathbf{i}_{\mathrm{m} s}^{\mathrm{g} \omega}, s \in \mathbb{N}_{0}$ enter from IMU with period $T_{q} \ll T_{o}$, and the measured values $\Lambda_{\mathrm{m} r}^{\mathrm{a}}$ enter from AS with period $T_{o}$ :

$\mathbf{i}_{\mathrm{m} s}^{\mathrm{g} \omega}=\operatorname{Int}\left(t_{s}, T_{q}, \boldsymbol{\omega}_{\mathrm{m}}^{\mathrm{g}}(\tau)\right)+\boldsymbol{\delta}_{s}^{\mathrm{n}} ; \boldsymbol{\Lambda}_{\mathrm{m} r}^{\mathrm{a}}=\boldsymbol{\Lambda}_{r} \circ \boldsymbol{\Lambda}_{r}^{\mathrm{n}} ; s \in \mathbb{N}_{0}$. Here measured vector $\boldsymbol{\omega}_{\mathrm{m}}^{\mathrm{g}}(t) \equiv(1+m) \mathbf{S}^{\Delta}\left(\boldsymbol{\omega}(t)+\mathbf{b}^{\mathrm{g}}\right)$ of SC angular rate is presented into the IMU base $\mathrm{G}$ taking into account the unknown small and slow variations of the IMU bias vector $\mathbf{b}^{\mathrm{g}}=\mathbf{b}^{\mathrm{g}}(t)$; orthogonal matrix $\mathbf{S}^{\Delta}(t)$ describes errors on a mutual angular position of the IMU and AS reference frames; scalar function $m=m(t)$ presents an unknown slow and small variation of the IMU scale factor, for example, $|m(t)| \leq 0.01$, when relation $1-m^{2} \cong 1$ is satisfied. We take into consideration the Gaussian noises $\boldsymbol{\delta}_{s}^{\mathrm{n}}$ and $\boldsymbol{\Lambda}_{k}^{\mathrm{n}}$ in the IMU and AS output signals. The problem consists in developing algorithms for obtaining the estimations $\hat{\boldsymbol{\Lambda}}_{l}, l \in \mathbb{N}_{0}$ with given period $T_{p}=t_{l+1}-t_{l}$ multiple to period $T_{o}$, in a general case, with a fixed delay $T_{d}$ with respect to the time moments $t_{r}$, and also in developing algorithms for the ADS calibration and alignment with the derivation of estimates $\hat{\mathbf{b}}_{r}^{\mathrm{g}}, \hat{\mathbf{S}}_{r}^{\Delta}$ and $\hat{m}_{r}$ during all modes of the SC attitude motion.

Principal problems get up on a planning the space land-survey and the SC angular guidance at its route motion when a space observation is executed at given time interval $t \in \mathrm{T}-$ determination of quaternion $\boldsymbol{\Lambda}^{p}(t)$, vectors of angular rate $\boldsymbol{\omega}^{p}(t)$ and acceleration $\varepsilon^{p}(t)$ in the form of explicit functions, proceed from the main requirement: optical image of the Earth given part must move by desired way at the telescope focal plane. Assume that for any time interval $\mathrm{T}$ we carried out the SC gui-dance attitude law by numerical integrating the quaternion kinematic equation in (4) and we have obtained numerical data in points $t_{l} \in \mathrm{T}$, $l \in \overline{\mathbb{N}} \subset \mathbb{N}_{0}$. This law corresponds to required scanning route $\boldsymbol{\Lambda}^{p}(t), \boldsymbol{\omega}^{p}(t)$ by arbitrary type - trace, orthodromic, with optimal equalization of a longitudinal IMV, stereo observation et al. The problem consists in analytical representation of the guidance law without any restriction on duration of interval $\mathrm{T}$.

If we have two adjacent SRs, then for the SC rotational maneuver (RM) we have obtained the boundary conditions by quaternion, vectors $\boldsymbol{\omega}$ and $\varepsilon$ and also by vector $\varepsilon^{*}$ in a time moment when the second SR is beginning. For the RM time interval $t \in \mathrm{T}_{\mathrm{p}}^{r} \equiv$ $\left[t_{\mathrm{i}}^{p}, t_{\mathrm{f}}^{p}\right], t_{\mathrm{f}}^{p} \equiv t_{\mathrm{i}}^{p}+T_{p}^{r}$ and the general boundary conditions

$$
\begin{aligned}
& \boldsymbol{\Lambda}\left(t_{\mathrm{i}}^{p}\right)=\boldsymbol{\Lambda}_{\mathrm{i}} ; \boldsymbol{\omega}\left(t_{\mathrm{i}}^{p}\right)=\boldsymbol{\omega}_{\mathrm{i}} ; \boldsymbol{\varepsilon}\left(t_{\mathrm{i}}^{p}\right)=\varepsilon_{\mathrm{i}} ; \\
& \boldsymbol{\Lambda}\left(t_{\mathrm{f}}^{p}\right)=\boldsymbol{\Lambda}_{\mathrm{f}} ; \boldsymbol{\omega}\left(t_{\mathrm{f}}^{p}\right)=\boldsymbol{\omega}_{\mathrm{f}} ; \boldsymbol{\varepsilon}\left(t_{\mathrm{f}}^{p}\right)=\boldsymbol{\varepsilon}_{\mathrm{f}} ; \boldsymbol{\varepsilon}^{*}\left(t_{\mathrm{f}}^{p}\right)=\varepsilon_{\mathrm{f}}^{*}
\end{aligned}
$$

taking into account given restrictions on vectors $\boldsymbol{\omega}(t)$ and $\boldsymbol{\varepsilon}(t)$ we consider the problem on synthesis of a guidance law at the spacecraft RM using analytic relations only.

At a land-survey SC lifetime up to 10 years its structure inertial and flexible characteristics are slowly changed in wide boundaries, the solar array panels are rotated with respect to the SC body and the communication antennas are pointing for information service. Therefore inertial matrix $\mathbf{A}^{\circ}$ and partial frequencies $\Omega_{j}^{q}$ of the SC structure oscillations in (1) are not complete certain. General problem consists in dynamical designing the GMC's robust digital control law $\mathbf{u}_{k}^{\mathrm{g}}=\left\{u_{p k}^{\mathrm{g}}\right\}$.

\section{SMOOTHING THE DISCRETE MEASUREMENTS}

The classical problem on polynomial approximation of the values $y_{s}=f\left(x_{s}\right), s=1 \div n$ for the unknown scalar function $y=f(x)$ 
as the polynomial $y=\sum_{i=0}^{m} a_{i} x^{i}$ with the degree $m<n$ using the method of least squares (MLS), consists in definition of the coefficients $a_{i}, i=0 \div m$ from the condition

$$
\sum_{s=1}^{n}\left\{\left(\sum_{i=0}^{m} a_{i} x_{s}^{i}\right)-y_{s}\right\}^{2} \Rightarrow \min .
$$

Using the elegant Gauss notation $[u] \equiv \sum_{s=1}^{n} u_{s}$, one can obtain the system of $m+1$ normal scalar equations

$$
\sum_{i=0}^{m} a_{i}\left[x^{i}\right]=[y] ; \sum_{i=0}^{m} a_{i}\left[x^{i+1}\right]=[x y] ; \ldots \sum_{i=0}^{m} a_{i}\left[x^{i+m}\right]=\left[x^{m} y\right] .
$$

At introducing column $\mathbf{a}=\left\{a_{0}, a_{1}, \ldots a_{m}\right\}$ this system is presented in the form $\mathbf{C a}=\mathbf{b}$ by the trivial way. Here matrix $\mathbf{C}=\left\|c_{i k}\right\|$ is symmetrical and "recursive" $\left(c_{i k}=c_{i-1, k+1}\right)$, and the required column a is computed on the basis of standard algorithms (Lanczos, 1956). For the MLS polynomial approximation the degree $m$ must be chosen taking into account the length of access data $y_{s}, s=1 \div n$. The solution of practical tasks demonstrates that it is rational to apply method (filter) of the Savitsky Goley (Orfanidis, 1996) polynomial smoothing that is a modification of the MLS for large values $n$. Here the sequence of the discrete values $y_{s}$ is approximated in a "moving" window (frame) with the length $n_{*} \ll n$, where $n_{*}$ is a whole odd number and also a "moving polynomial" with small degree $m$, for example $m=3$. The first frame is formed by the values $y_{s}=f\left(x_{s}\right)$, $s=1 \div n_{*}$ beginning from the first measurement, and a polynomial with the given degree is constructed for it by the MLS. Then the frame is displaced on one value and the approximation is carried out again. Every time in the output sequence one can use only the single value of an approximation polynomial that corresponds to the center $\left(n_{*}-1\right) / 2$ of the current position of a "moving frame". The values of 3-dimensional vector function $\mathbf{y}_{s}=\mathbf{f}\left(x_{s}\right)$ of the scalar argument using the Savitsky - Goley filter are smoothed out by application of this procedure for values of each component of the vector composed from mapping values of the vector function on the axes of some orthogonal basis.

The problem on definition of the mutual orientation of two orthogonal bases on the basis of the data about two sets of the unit vectors that are arbitrarily placed in the bases, is more complex. Let a set of the units $\mathbf{b}_{i}$ be given that are measured in the base $\mathbf{B}$, and a set of the units $\mathbf{r}_{i}$ corresponding to them specified in the base $\mathbf{I}$. The classical problem of vector matching problem) is formulated as follows: let us define an orthogonal matrix $\mathbf{A}$ with a determinant equal to +1 , which minimizes the quadratic index

$$
L(\mathbf{A})=\frac{1}{2} \Sigma a_{i}\left|\mathbf{b}_{i}-\mathbf{A} \mathbf{r}_{i}\right|^{2} \Rightarrow \min ,
$$

where the numbers $a_{i}>0$ are the weighing coefficients. It has been strictly proved that the solution of this problem is the optimal quaternion $\boldsymbol{\Lambda}$ that is equivalent to the required orthogonal matrix $\mathbf{A}$ and is defined as an eigenvector of the matrix $\mathbf{K}$ with the maximum eigenvalue $q_{\max }$, e. g. by relations

$$
\begin{gathered}
\mathbf{z}=\Sigma a_{i} \mathbf{b}_{i} \times \mathbf{r}_{i} ; \quad \mathbf{B}=\Sigma a_{i} \mathbf{b}_{i} \mathbf{r}_{i}^{\mathrm{t}} ; \quad \mathbf{S}=\mathbf{B}+\mathbf{B}^{\mathrm{t}} ; \\
\mathbf{K}=\left[\begin{array}{cc}
\operatorname{tr} \mathbf{B} & \mathbf{z}^{\mathrm{t}} \\
\mathbf{z} & \mathbf{S}-\mathbf{I}_{3} \operatorname{tr} \mathbf{B}
\end{array}\right] ; \quad \mathbf{K} \boldsymbol{\Lambda}=q_{\max } \mathbf{\Lambda} .
\end{gathered}
$$

Relations (7) represent the QUEST algorithm (Markley and Mortar, 2000), that is further applied for processing the measuring information obtained both in the mode of astronomical checking axes' concordance (ACAC), see Fig. 6, and in the mode of marked checking axes' concordance (MCAC), see Fig. 7. The quaternion $\Lambda$ is an one-one related to the MRP vector $\sigma$ by the explicit analytic relations, that permits a transforming the problem on smoothing the quaternion data to standard task on smoothing the vector measurements.

\section{DEFINITION OF THE TELESCOPE ORIENTATION}

In the ACAC mode at scanning the star sky with the angular rate $\omega_{z}^{\star} \approx 0.015 \mathrm{deg} / \mathrm{s}$ on the SC pitch channel, the "moving window" is organizing with telescope's field-of-view at a fixed frequency of accumulating the electronic image charge packets along the columns of the CCD matrix. At first, we define the sequence of quaternion $\boldsymbol{\Lambda}_{s}^{\mathrm{s}}$ for the base $\mathbf{S}$ with exact binding to the time moments $t_{s}, s \in \mathbb{N}_{0}$, on the star sky photo. Then two sets of the unit directions on stars are defined for each frame: the set of the unit vectors $\mathbf{r}_{\nu}^{\mathrm{s}}$ in the VRF by the stars' relative coordinates into the CCD matrix and the set of unit vectors $\mathbf{b}_{\nu}^{\mathrm{s}}$ in IRF by direct ascents $\alpha_{\nu}$ and inclinations $\delta_{\nu}, \nu=1 \div n$ for stars from the star catalogue FK-5. In completion the QUEST procedure is called to determine the SRF attitude quaternion $\Lambda_{i}^{\mathrm{s}}$ values with respect to the IRF at the time moments $t_{i}^{\mathrm{m}}, i=1 \div N_{k}$, where $N_{k}$ is the frame quantity in the electronic photo. As a result, one can obtain the sequences of values both quaternion $\boldsymbol{\Lambda}_{s}^{\mathrm{v}}$ and quaternion $\boldsymbol{\Lambda}_{s}^{\mathrm{s}}$ for the RFs orientation in the IRF. For describing deviation of the VRF from its required position in the IRF we applied kinematic parameters in the form of angle $\delta \phi_{\mathrm{e}}$ (deviation of unit $\mathbf{v}_{1}$ from its required position) and angle $\delta \phi_{\mathrm{x}}$ (a turn about a telescope's optical axis). For these parameters we have studied the RMS deviations obtained with the frame dimension $1.3 \times 1.3 \mathrm{deg}$. Results testify that to ensure a permissible error on determination of the telescope axis' actual position into the IRF, it is enough ten stars being observed in the frame. The error $\delta \phi_{\mathrm{x}}$ is dozen times worse even at larger star's quantity. That result is explained by the small telescope's field-of-view, e. g. by the insufficient measuring base. The elaborated technique for a more accurate definition is based on widening the measuring astronomical basis at the expense of long-term SC scanning motion, perhaps with the time technology breaks of star observation by a telescope: it is assumed the possibility of the telescope motion with the closed cover (Somov et al., 2008). We have carried out numerical calculations with filtering of the telescope attitude estimations by Savitsky - Goley method. The obtained results indicated that such technique ensures the RMS deviation on angle $\delta \phi_{\mathrm{x}}$ no more than 1 arc sec.

In the MCAC mode a definition of the telescope orientation actual values $\boldsymbol{\Lambda}_{s}^{\mathrm{s}}$ is carried out by a scanning optoelectronic observing the Earth polygons with terrestrial marked objects, see Fig. 7. Here also only the measuring information from the telescope is applied. In this mode the SC fulfills a program motion, given by a set of splines for the MRP vector $\boldsymbol{\sigma}(t)$, during the ACS operation. These vector splines are calculated from the conditions of observing a terrestrial polygon with given azimuth of scanning. Sequence of actual VRF angular positions in the IRF at observing a terrestrial polygon is carried out by well-known method of backward dynamical photogrammetric intersection with applying the precise tie to the time moments $t_{s}$ for appearing the electronic images of the polygon's marked objects on the electronic photoframe. Here we apply our technique (Somov and Butyrin, 2012) that is similar to the technique for ACAC mode presented above.

\section{DEFINITION OF THE STS ORIENTATION}

The base $\mathbf{A}=\left\{\mathbf{a}_{1}, \mathbf{a}_{2}, \mathbf{a}_{3}\right\}$ of the STC, see Fig. 4, is calculated by processing an accessible measuring information, obtained at both the ACAC and MCAC modes from any combination of the STs. The CCD matrix in the focal plane of each ST is fixed in the BRF, therefore, the "summary" field-of-view for the STC, based on any combination from no smaller then two star trackers, puts together a large measuring base. This measuring base is quite 


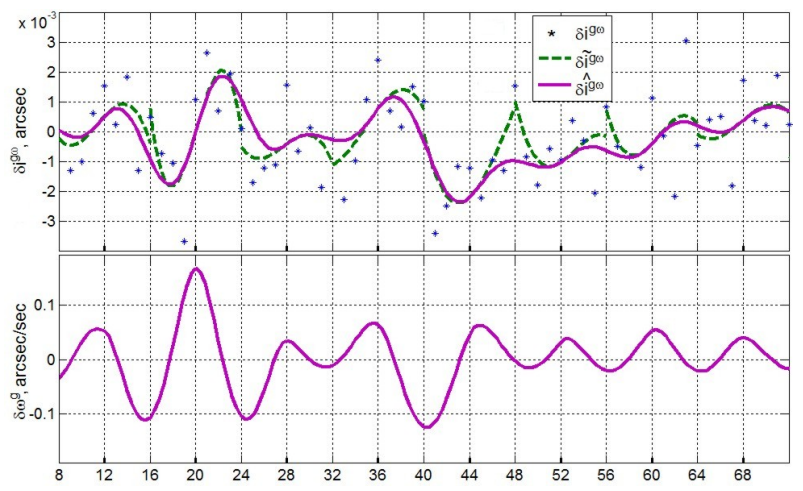

Figure 8. Errors of a filtering by the two-pass technology

sufficient for high-accuracy determination of the STCRF angular position in the same inertial base I. Naturally, the best results are obtained if it is possible to obtain measuring information from all forth STs and to fulfill next onboard processing, first by the QUEST algorithm and then with filtering by Savitsky - Goley method. The necessity of additional alignment verification in the MCAC mode is accounted by different conditions of observing the "cold" space and the "warm" Earth by the telescope. In the other limited calculated case, the virtual STCRF is constructed based on the information about angular positions of the optical axes units $\mathrm{a}_{p}$ for any two STs by well-known TRIAD algorithm.

\section{ALIGNING THE TELESCOPE WITH THE STC}

It is clear, that if we have estimations on the VRF and STCRF orientation in the same inertial base $\mathbf{I}$, then it is simple to obtain a constant correction quaternion for taking into account their reciprocal position. Such a correction quaternion is applied in the SC attitude control system at observing the Earth surface. After fulfilling an alignment verification on terrestrial polygons, the $\mathrm{SC}$ onboard equipment has the possibility for operative solution of tasks on a posteriori restore of actual the VRF attitude at any time moment of the Earth's scanning optoelectronic observation. Initial alignment of a telescope and the virtual STCRF is fulfilled during a time period of the SC in-flight tests on terrestrial polygons, where the marked objects' coordinates are known with fine accuracy. At next regular exploitation of the land-survey SC there is needed from time to time to fulfill an observing some passing parts of the Earth surface in small neighbourhood of the SC trace, for which the place maps have known coordinates of conditional marked objects, for example maps of large cities.

\section{THE ADS CALIBRATION AND ALIGNMENT}

Suggested principal ideas are as follows: (i) there is needed to define estimations $\hat{\mathbf{S}}^{\Delta}$ and $\hat{m}$ only on the whole for virtual bases $A$ and $\mathrm{G}$ with respect to main base $\mathbf{S}=\mathbf{B}$, without concrete details on errors of individual onboard measuring devices and to integrate the kinematic equations with a small computing drift; (ii) an idea is being developed to use approximation and interpolation of the measured information in the intermediate points with period $T_{q}$ multiple to the main sampling period $T_{\mathrm{o}}$; (ii) identification of the IMU drift vector $\mathbf{b}^{\mathrm{g}}$ is ensured by nonlinear discrete Luenberger observer. We provide a forming of estimations $\hat{\mathbf{b}}_{r}^{\mathrm{g}}$, $\hat{\mathbf{S}}_{r}^{\Delta}$ and $\hat{m}_{r}$ fixed on period $T_{o}$ when estimations $\hat{\mathbf{b}}_{r}^{\mathrm{g}}$ is updated on-line, and estimations $\hat{\mathbf{S}}_{r}^{\Delta}, \hat{m}_{r}$ are regularly formed off-line, i.e. thier are based on the processing of available measurement data, accumulated during long-term time intervals.

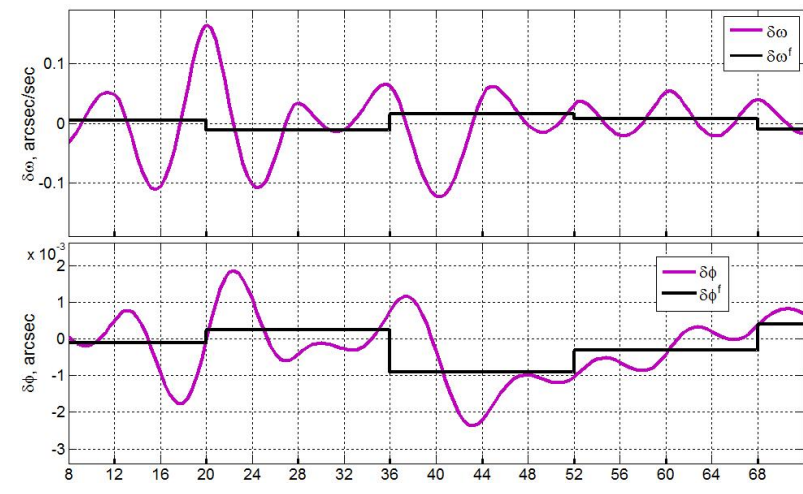

Figure 9. Errors on filtering of the IMU output signals

For discrete filtering the measured values of the quasi-coordinate increment vector $\mathbf{i}_{\mathrm{ms}}^{\mathrm{g} \omega}$ we used the two-pass filtering technology (Somov et al., 2017) - combination of approximation of the data $\mathbf{i}_{\mathrm{m} s}^{\mathrm{g} \omega}$ by the vector polynomial $\tilde{\mathbf{i}}_{\mathrm{m} r}^{\mathrm{g} \omega}(\tau)$ of 3 rd order in sliding window with 9 measurements on the MLS and the spline interpolation on centers of two adjacent sliding windows by spline $\check{\mathbf{i}}_{\mathrm{m} r}^{\mathrm{g} \omega}(\tau)$ of 5th order for local time $\left.\tau=t-r T_{o} \in\left[0, T_{o}\right]\right)$. The technology is illustrated by scheme in Fig. 8. Here errors $\delta \mathbf{i}^{\mathrm{g} \omega}$ of measured quasi-coordinate are marked by the "stars" for time moments $t_{s}$ (index $s$ is shown only), green dotted lines are given polynomials $\tilde{\mathbf{i}}^{g \omega}(\tau)$ of 3rd order and burgundy line is presented the smoothly conjugate splines $\hat{\mathbf{i}}^{\mathrm{g} \omega}(\tau)$ of 5th order. Error $\delta \boldsymbol{\omega}^{\mathrm{g}}(\tau)$ for estimation $\hat{\omega}^{\mathrm{g}}(\tau)$ on the angular rate is presented in lower part of the figure. The estimation $\hat{\omega}^{\mathrm{g}}(\tau)$ strongly agreed with estimation $\hat{\mathbf{i}}^{\mathrm{g} \omega}(\tau)$ as it is carried out by explicit analytical relations. At compensation of errors on the drift vector, a mutual angular position of the IMU and AS reference frames and on a scale factor, the continuous vector estimation $\hat{\mathbf{i}}_{r}^{\omega}(\tau)$ in base $\mathrm{A}$ is computed by relation $\hat{\mathbf{i}}_{r}^{\omega}(\tau)=\left(1-\hat{m}_{r}\right)\left(\hat{\mathbf{S}}_{r}^{\Delta}\right)^{\mathrm{t}}\left(\check{\mathbf{i}}_{r}^{\mathrm{g} \omega}(\tau)-\hat{\mathbf{b}}_{r}^{\mathrm{g}} \tau\right)$ on $r$-th time interval $\mathrm{T}_{r} \equiv\left[t_{r}, t_{r+1}\right]$, moreover $\hat{\mathbf{i}}_{r+1}^{\omega}=\hat{\mathbf{i}}_{r}^{\omega}\left(T_{o}\right)$.

Identification of IMU bias $\mathbf{b}^{\mathrm{g}}$ is carried out with period $T_{o}$ by extended Luenberger filter (ELF). At the time interval $\mathrm{T}_{r}$ an estimation of the SC attitude is attained by integration of the vector differential equation $\dot{\hat{\boldsymbol{\sigma}}}_{r}(\tau)=\mathbf{F}^{\sigma}\left(\hat{\boldsymbol{\sigma}}_{r}(\tau), \hat{\boldsymbol{\omega}}_{r}(\tau)\right)$ (5) using ODE45 method (Shampine, 1986) with a forming of an estimation of the MRP vector $\hat{\boldsymbol{\sigma}}_{r}(\tau)$. For this vector equation an initial condition is calculated by signals of the ELF. Assume that at the time moment $t=t_{r}$ we have the AS information on the SC orientation in the form of quaternion $\Lambda_{\mathrm{m} r}^{\mathrm{a}}$, the correcting vector $\Delta \mathbf{p}_{r}\left(g_{2}^{\mathrm{o}}, \mathbf{Q}_{r}\right)$ and quaternion $\Delta \mathbf{P}_{r}\left(g_{1}^{\mathrm{o}}, \mathbf{Q}_{r}\right)$ were formed, where $\mathbf{Q}_{r} \equiv\left(q_{0 r}, \mathbf{q}_{r}\right) \equiv\left(C_{\frac{\varphi_{r}}{2}}, \mathbf{e}_{r}^{q} S_{\frac{\varphi_{r}}{2}}\right) \equiv \mathbf{Q}_{k}\left(\mathbf{e}_{k}^{q}, \varphi_{k}\right)=\tilde{\boldsymbol{\Lambda}}_{\mathrm{m} r}^{\mathrm{a}} \circ \hat{\boldsymbol{\Lambda}}_{r}$. At the same time moment $t_{r}$ initial condition $\hat{\boldsymbol{\sigma}}_{r}(0) \equiv \hat{\boldsymbol{\sigma}}_{r}$ is defined by transformation $\hat{\Lambda}_{r} \Rightarrow \hat{\boldsymbol{\sigma}}_{r}$ for calculation of the estimate $\hat{\boldsymbol{\sigma}}_{r}(\tau)$ on the $r$-th interval. After such integration one can obtain the MRP vector's value $\hat{\boldsymbol{\sigma}}_{r+1}=\hat{\boldsymbol{\sigma}}_{r}\left(T_{o}\right)$ and the value of quaternion $\hat{\mathbf{R}}_{r}$ is calculated by transformation $\hat{\boldsymbol{\sigma}}_{r+1} \Rightarrow \hat{\mathbf{R}}_{r}$. The developed nonlinear ELF has the form (Somov et al., 2017)

$$
\begin{aligned}
& \hat{\mathbf{\Lambda}}_{r+1}=\hat{\mathbf{R}}_{r} \circ \Delta \mathbf{P}_{r}\left(g_{1}^{\mathrm{o}}, \mathbf{Q}_{r}\right) ; \hat{\mathbf{b}}_{r+1}^{\mathrm{g}}=\hat{\mathbf{b}}_{r}^{\mathrm{g}}+\Delta \mathbf{p}_{r}\left(g_{2}^{\mathrm{o}}, \mathbf{Q}_{r}\right) ; \\
& \Delta \mathbf{P}_{r+1}=\mathbf{Q}_{r+1}\left(\mathbf{e}_{r+1}^{q}, g_{1}^{\mathrm{o}} \varphi_{r+1}\right) ; \Delta \mathbf{p}_{r+1}=4 g_{2}^{\mathrm{o}} \boldsymbol{\sigma}_{r+1}^{q},
\end{aligned}
$$

where both the quaternion and vector relations are applied. Moreover, the MRP vector $\boldsymbol{\sigma}_{r+1}^{q}$ is defined analytically on the quaternion value $\mathbf{Q}_{r+1}$, and the ELF scalar coefficients $g_{1}^{\circ}, g_{2}^{\circ}$ are calculated by explicit analytical relations. In final stage the MRP vector values $\boldsymbol{\sigma}_{s}$ are processed by recurrent discrete filter with period $T_{p}$. The filtering technology is illustrated by scheme in Fig. 9. Here $\delta \omega(t), \delta \phi(t)$ are the continuous mismatches and 
their filtered digital values $\delta \omega_{l}^{\mathrm{f}}, \delta \phi_{l}^{\mathrm{f}}$ are presented by black lines when period $T_{p}=16 T_{q}$. As a result, one can obtain the MRP vector values $\hat{\boldsymbol{\sigma}}_{l}$ which are applied for a forming of the quaternion estimate $\hat{\Lambda}_{l}, l \in \mathbb{N}_{0}$ with given period $T_{p}$ using transformation $\hat{\boldsymbol{\sigma}}_{l} \Rightarrow \hat{\boldsymbol{\Lambda}}_{l}$. The IMU astronomical correction is temporarily disabled when module $\omega(t)$ of the SC angular rate vector satisfies inequality $\omega(t) \geq \omega_{1}^{\mathrm{m}}=1 \mathrm{deg} / \mathrm{s}$ during a time interval of the SC rotational maneuver, but estimation of the $\mathrm{SC}$ angular position continues using forecast of the $\hat{\mathbf{b}}^{\mathrm{g}}$ variation. For the direct account of the AS measurement noise, the observer of this structure may be presented by the extended Kalman filter (EKF). Moreover, the constant covariance and gain matrices are determined analytically only for the steady state of EKF operation. Here for identifying drift vector $\mathbf{b}^{\mathrm{g}}$ taking into account dependence $\sigma^{\mathrm{a}}(\omega)$ for the RMS deviation, we need to solve numerically the Riccati matrix equation. In these circumstances, it is reasonable to apply the observer of the IMU drift in the ELF form assigning its parameters so that to ensure the quality of estimation $\hat{\mathbf{b}}^{\mathrm{g}}$ to be close to the quality of the EFK with a constant value $\sigma^{\mathrm{a}}=\sigma^{\mathrm{a}}\left(\omega_{1}^{\mathrm{m}}\right)$.

\section{PLANNING OF AN AREA LAND-SURVEY}

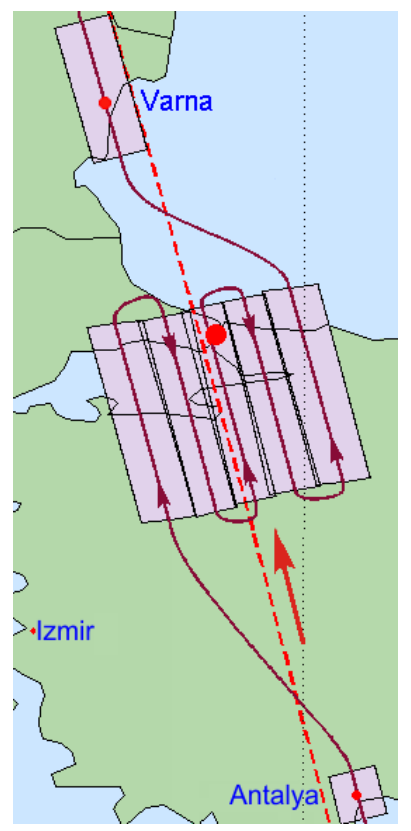

Figure 10. The area imagery
The aim of an area land-survey is to cover a given area on the Earth's surface with geographical center $C\left(L_{c}, B_{c}, H_{c}\right)$ by a sequence of partly overlapping scanning routes (OSRs). Assume that optoelectronic converters (OECs) in the telescope FP have the reverse mode. The initial data for planning such a land-survey are the size of the area $S=a \times b$ with length $a$ and width $b$, parameters of the $\mathrm{SC}$ orbital motion, characteristics of the telescope and OECs, restrictions on kinematic parameters of the SC angular motion. The values of azimuth deviation of orthodromic OSRs from the route are up to $\pm \pi / 9$ and $(1 \pm(1 / 9)) \pi$. The main aspect in solving this problem consists in determining required number of scans $N$ and longitudinal IMV in the telescope FP during the OSR performing. Next this information is applied for synthesis of the SC guidance laws at runnig both the central and side scans.

Central scan (CS) is the one, which center coincides with center $C$ of the area, and the ORF plane $y^{\circ} \mathrm{O} z^{\circ}$ at the scanning time moment $t_{c}$ crosses point $C$. Estimated number of scans is: $N=$ $2 b(1-p / 50) /\left(s_{0}+s_{m}\right)$, where $s_{0}$ and $s_{m}$ are the sizes of the projections of the OEC central line on the Earth's surface with minimum (at the time moment $t_{c}$ ) and maximum distance from center $C$ of the area, $p \in[5,10]$ - percentage of overlap of the scans. The maximum distance corresponds to the case with restrictions of pitch angle or of observation distance $D$. For conditional CS the initial forecast of required longitudinal IMV $V_{c}^{i}$ is carried out by the trace observation scheme. Moreover, we obtain initial estimation of duration of the scan $T_{c}=2 a f_{e} /\left(D V_{c}^{i}\right)$, where $f_{e}$ is effective focal length of the telescope, and the duration of areal land-survey is $T_{a}=N T_{c}+(N-1) T_{r}$, where $T_{r}=T_{c} / 3$ is

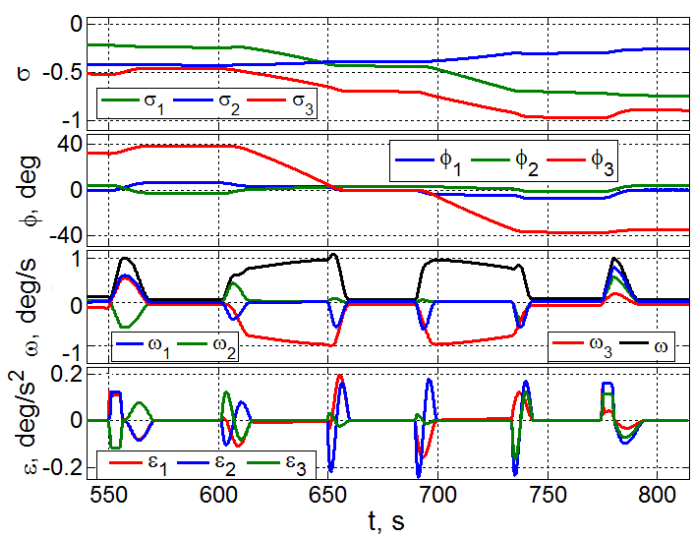

Figure 11. The SC spline guidance law for areal land-survey

predicted duration of the satellite RM between partly overlapping scanning routes. We determine geodetic coordinates of the begin$\operatorname{ning} C_{\mathrm{i}}$ and end $C_{\mathrm{f}}$ of the central scan equidistant from the point $C$ on the value of $a / 2$ with azimuth $A$ in forward and opposite directions at time moments $t_{c \mathrm{i}}=t_{c}-T_{c} / 2$ and $t_{c \mathrm{f}}=t_{c}+T_{c} / 2$, accordingly. Then the values $t_{c \mathrm{i}}, t_{c \mathrm{f}}, V_{c}^{i}, T_{c}$ and azimuth $A$ are specified by iterative method using a numerical simulation of the SC spatial motion when performing the orthodromic OSR at time interval $t \in\left[t_{c \mathrm{i}}, t_{c \mathrm{f}}\right]$. As a result, we provide the allowable deviation of the CS length from the specified value and obtain the characteristics of the CS on the Earth's surface: length $a_{c}$ and width $d_{c}$ at the OEC center, the coverage area, the CS beginning and end time moments, the geodetic coordinates of the center and of corner points in a contour of the arbitrary central scan. The orthodromic OSR adjacent to the arbitrary CS is called the side scan (SS). The calculation of the SS is similar, but there are additional iterations in order to assign the position of its center $C_{b}$. The initial coordinates of center $C_{b}$ are determined by moving on the Earth's surface from point $C$ at the distance $d^{i}= \pm \Delta d \Delta n / N$ with azimuth $A \pm \pi / 2$. Here the symbols $(+)$ and $(-)$ correspond to the right and left SSs for the SC flight, $\Delta d=d_{c}^{m}-d_{c}$ represents the difference between width $d_{c}$ of the central scan and its width $d_{c}^{m}$, calculated at the maximum distance, $\Delta n$ is the difference in modulus between the current side and central scans. Estimation of time moment $t_{b c}$ for scanning of center $C_{b}$ is the following: $t_{b c}=t_{c}+T_{c}+T_{r}$. We assign the initial value of the SS longitudinal IMV in the form of $V_{b c}^{i}= \pm V_{c}^{i} / 2$, where the symbols $(+)$ and $(-)$ correspond to odd and even numbers of such scans. Then the IMV values and other SS parameters are iteratively refined. In the synthesis of the subsequent SSs, all calculations are carried out with samples gained from the previous $\mathrm{SS}$, which performs as the central scan. If the number of scans is odd, then the central conditional and the actual scans are the same. For even $N$ the position of the actual scan center displaced on a distance of $d_{c} / 2$ with azimuth $A-\pi / 2$, and the time moment of its scanning is changed by the value $\Delta t_{c}=-\left(T_{c}+T_{r}\right) / 2$. Moreover, the area center $C$ will be located in the overlap of central parts of two scans.

Fig. 10 represents the map with projections of scans and of telescope target line trace obtained in planning two single SRs and areal land-surveying neighborhoods of Istanbul for the SC in sun-synchronous orbit with altitude of $720 \mathrm{~km}$ and inclination of $98.27 \mathrm{deg}$, when the allowed deviation of the target line from Nadir is within the cone with semi-angle of $40 \mathrm{deg}$. The first SR Antalya, with duration of $10 \mathrm{~s}$, starts at the point with coordinates of $N 36.68 \mathrm{deg}, E 30.65 \mathrm{deg}$ and runs with alignment of longitudinal IMV. On the Earth's surface the scanning route 
has a length of $54.78 \mathrm{~km}$ and a width of $46.87 \mathrm{~km}$. Further the areal land-survey is performed using five orthodromic OSRs with rotational maneuvers in-between. Moreover, the scanning area of the Earth's surface has dimension $200 \times 203 \mathrm{~km}$, geodetic coordinates of its center are $N 40.5 \mathrm{deg}, E 29.2 \mathrm{deg}$. The final SR Varna for trace observation, with duration of $20 \mathrm{~s}$, starts at the point with coordinates $N 43.21$ deg and $E 27.9$ deg. On the Earth's surface this SR has a length of $135.92 \mathrm{~km}$ and a width of $48.75 \mathrm{~km}$.

\section{THE SPACECRAFT ATTITUDE GUIDANCE LAWS}

Analytic matching solution have been obtained for problem of the SC guidance during any scanning route. The solution is based on a vector composition of all motions in GRF using the following reference frames: HRF, SRF and FRF. For any observed point $\mathrm{C}$ the oblique range $\mathrm{D}$ is analytically calculated as $\mathrm{D}=\left|\mathbf{r}_{\mathrm{c}}^{\mathrm{e}}-\mathbf{r}^{\mathrm{e}}\right|$. If matrix $\mathbf{C}_{\mathrm{h}}^{\mathrm{s}} \equiv \tilde{\mathbf{C}}=\left\|\tilde{c}_{i j}\right\|$ defines the SRF orientation in HRF $\mathbf{E}_{\mathrm{e}}^{\mathrm{h}}$, then for any point $\mathrm{M}\left(\tilde{y}^{i}, \tilde{z}^{i}\right)$ in the telescope FP the components $\tilde{V}_{y}^{i}$ and $\tilde{V}_{z}^{i}$ of the IMV normed vector are computed as follows

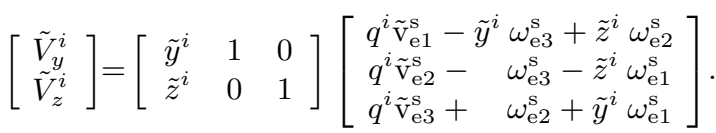

Here $\tilde{y}^{i}=y^{i} / f_{e}, \tilde{z}^{i}=z^{i} / f_{e}$ are normed focal coordinates where function $q^{i} \equiv 1-\left(\tilde{c}_{21} \tilde{y}^{i}+\tilde{c}_{31} \tilde{z}^{i}\right) / \tilde{c}_{11}$, and vector of normed SC's mass center velocity has components $\tilde{\mathrm{v}}_{\mathrm{e} i}^{\mathrm{s}}=\mathrm{v}_{\mathrm{e} i}^{\mathrm{s}} / \mathrm{D}, i=1 \div 3$. Further, ratio (9) is applied for calculation of the SC guidance law at any scanning route.

Consider the time interval $\mathrm{T} \equiv[0, T]$ with the following notations for its four points $\tau_{p}, p=1 \div 4: \tau_{1}=0, \tau_{2}=T / 3$, $\tau_{3}=2 T / 3$ and $\tau_{4}=T$. For six values $\boldsymbol{\omega}_{l}=\boldsymbol{\omega}\left(t_{l}\right)$ nearby points $\tau_{1}=0, \tau_{4}=T$ standard interpolation is carried out by the vector spline of degree five. This allows us to calculate values $\varepsilon_{1}=\dot{\boldsymbol{\omega}}\left(\tau_{1}\right)$ and $\varepsilon_{4}=\dot{\boldsymbol{\omega}}\left(\tau_{4}\right)$ of angular acceleration vector. For four points $\tau_{p} \in \mathrm{T}$ values $\sigma_{p}, p=1 \div 4$ are computed, also values $\dot{\boldsymbol{\sigma}}_{p}$ and $\ddot{\boldsymbol{\sigma}}_{p}, p=1,4$ for two boundary points. Interpolation of the RMP vector $\boldsymbol{\sigma}(t) \forall t \in \mathrm{T}$ is carried out by the vector spline of 7 degree $\boldsymbol{\sigma}_{a}(t)=\sum_{0}^{7} \mathbf{a}_{s} t^{s}$ with 8 columns $\mathbf{a}_{s} \in \mathbb{R}^{3}, s=0 \div 7$ of unknown coefficients. Eight columns $\mathbf{a}_{s}$ are defined for spline $\boldsymbol{\sigma}_{a}(t)$ on the basis of (i) three boundary conditions $\boldsymbol{\sigma}_{a}(0)=\boldsymbol{\sigma}_{1} ; \dot{\boldsymbol{\sigma}}_{a}(0)=\dot{\boldsymbol{\sigma}}_{1} ; \ddot{\boldsymbol{\sigma}}_{a}(0)=\ddot{\boldsymbol{\sigma}}_{1}$ on the left end of interval $\mathrm{T}$, which results in $\mathbf{a}_{0}=\boldsymbol{\sigma}_{1}, \mathbf{a}_{1}=\dot{\boldsymbol{\sigma}}_{1}$ and $\mathbf{a}_{2}=\ddot{\boldsymbol{\sigma}}_{1} / 2$; (ii) two conditions $\boldsymbol{\sigma}_{a}\left(\tau_{2}\right)=\boldsymbol{\sigma}_{2} ; \boldsymbol{\sigma}_{a}\left(\tau_{3}\right)=\boldsymbol{\sigma}_{3}$ in points $\tau_{2}$ and $\tau_{3}$; (iii) three boundary conditions $\sigma_{a}(T)=\sigma_{4}$; $\dot{\boldsymbol{\sigma}}_{a}(T)=\dot{\boldsymbol{\sigma}}_{4} ; \ddot{\boldsymbol{\sigma}}_{a}(T)=\ddot{\boldsymbol{\sigma}}_{4}$. Elaborated matrix relation is applied for simultaneous analytical computation of all five sought columns $\mathbf{a}_{s}, s=3 \div 7$.

For $\mathrm{SC}$ rotational maneuver on a time interval $\mathrm{T}_{\mathrm{p}}^{r}$ with the general boundary conditions (6) we have developed analytical method for synthesis of the SC angular guidance law based on the necessary and sufficient condition for solvability of Darboux problem. Here the solution is presented as the result of composition by three simultaneously derived rotations of "embedded" bases $\mathbf{E}_{k}$ about the unit vectors $\mathbf{e}_{k}, k=1 \div 3$ of Euler axes, quaternion $\boldsymbol{\Lambda}$ is defined as $\boldsymbol{\Lambda}(t)=\boldsymbol{\Lambda}_{\mathrm{i}} \circ \boldsymbol{\Lambda}_{1}(t) \circ \boldsymbol{\Lambda}_{2}(t) \circ \boldsymbol{\Lambda}_{3}(t)$, where $\boldsymbol{\Lambda}_{k}(t)=\left(\cos \left(\varphi_{k}(t) / 2\right), \mathbf{e}_{k} \sin \left(\varphi_{k}(t) / 2\right)\right)$ and $\varphi_{k}(t)$ is angle of $k$ 's rotation. Let us quaternion $\boldsymbol{\Lambda}^{*} \equiv\left(\lambda_{0}^{*}, \boldsymbol{\lambda}^{*}\right)=\tilde{\boldsymbol{\Lambda}}_{\mathrm{i}} \circ \boldsymbol{\Lambda}_{\mathrm{f}}$ has unit vector $\mathbf{e}_{3}=\boldsymbol{\lambda}^{*} / \sin \left(\varphi^{*} / 2\right)$ of 3 rd rotation with computed angle $\varphi^{*}=2 \arccos \left(\lambda_{0}^{*}\right)$. For quaternions $\boldsymbol{\Lambda}_{k}$ the boundary conditions

$$
\boldsymbol{\Lambda}_{1}\left(t_{\mathrm{i}}^{p}\right)=\boldsymbol{\Lambda}_{1}\left(t_{\mathrm{f}}^{p}\right)=\boldsymbol{\Lambda}_{2}\left(t_{\mathrm{i}}^{p}\right)=\boldsymbol{\Lambda}_{2}\left(t_{\mathrm{f}}^{p}\right)=\mathbf{1} ;
$$$$
\boldsymbol{\Lambda}_{3}\left(t_{\mathrm{i}}^{p}\right)=\mathbf{1}, \boldsymbol{\Lambda}_{3}\left(t_{\mathrm{f}}^{p}\right)=\left(\cos \left(\varphi_{3}^{\mathrm{f}} / 2\right), \mathbf{e}_{3} \sin \left(\varphi_{3}^{\mathrm{f}} / 2\right)\right)
$$

are applied, where $\varphi_{3}^{\mathrm{f}}=\varphi^{*}$ and $\mathbf{1}$ is the unit quaternion. We use notations $\boldsymbol{\omega}^{(k)}, \boldsymbol{\varepsilon}^{(k)}, \dot{\boldsymbol{\varepsilon}}^{(k)}$ with $k=1 \div 3$ for vectors $\boldsymbol{\omega}, \boldsymbol{\varepsilon}$ and $\dot{\boldsymbol{\varepsilon}}$ in base $\mathbf{E}_{k}$ and the vector operator $\mathbf{a}_{k-1}^{(k)}=\mathbf{\Phi}\left(\mathbf{a}_{k-1}, \boldsymbol{\Lambda}_{k}\right) \equiv \tilde{\boldsymbol{\Lambda}}_{k}$ 。 $\mathbf{a}_{k-1} \circ \boldsymbol{\Lambda}_{k}$ for conversion from basis $\mathbf{E}_{k-1}$ to basis $\mathbf{E}_{k}$. Assume that we assigned vectors $\boldsymbol{\omega}_{1}(t)=\dot{\varphi}_{1}(t) \mathbf{e}_{1}, \boldsymbol{\varepsilon}_{1}(t)=\ddot{\varphi}_{1}(t) \mathbf{e}_{1}$ and $\dot{\boldsymbol{\varepsilon}}_{1}(t)=\dddot{\varphi}_{1}(t) \mathbf{e}_{1}$. Then vectors $\boldsymbol{\omega}(t), \boldsymbol{\varepsilon}(t)$ and $\dot{\boldsymbol{\varepsilon}}(t)$ in the BRF are computed by the recurrent formulas with $k=2,3$ :

$\boldsymbol{\omega}_{k-1}^{(k)}=\boldsymbol{\Phi}\left(\boldsymbol{\omega}_{k-1}, \boldsymbol{\Lambda}_{k}\right) ; \boldsymbol{\varepsilon}_{k-1}^{(k)}=\boldsymbol{\Phi}\left(\boldsymbol{\varepsilon}_{k-1}, \boldsymbol{\Lambda}_{k}\right) ; \dot{\boldsymbol{\varepsilon}}_{k-1}^{(k)}=\boldsymbol{\Phi}\left(\dot{\boldsymbol{\varepsilon}}_{k-1}, \boldsymbol{\Lambda}_{k}\right) ;$ $\boldsymbol{\omega}^{(k)}=\boldsymbol{\omega}_{k-1}^{(k)}+\boldsymbol{\omega}_{k} ; \quad \boldsymbol{\varepsilon}^{(k)}=\boldsymbol{\varepsilon}_{k-1}^{(k)}+\boldsymbol{\varepsilon}_{k}+\boldsymbol{\omega}_{k-1}^{(k)} \times \boldsymbol{\omega}_{k} ;$ $\dot{\boldsymbol{\varepsilon}}^{(k)}=\dot{\boldsymbol{\varepsilon}}_{k-1}^{(k)}+\dot{\boldsymbol{\varepsilon}}_{k}+\boldsymbol{\omega}_{k-1}^{(k)} \times \boldsymbol{\varepsilon}_{k}+\left(2 \boldsymbol{\varepsilon}_{k-1}^{(k)}+\boldsymbol{\omega}_{k-1}^{(k)} \times \boldsymbol{\omega}_{k}\right) \times \boldsymbol{\omega}_{k}$. As a result, we obtain functions $\boldsymbol{\omega}(t)=\boldsymbol{\omega}^{(3)}(t), \boldsymbol{\varepsilon}(t)=\boldsymbol{\varepsilon}^{(3)}(t)$ and $\varepsilon^{*}(t)=\dot{\varepsilon}(t)=\dot{\boldsymbol{\varepsilon}}^{(3)}(t)$ by explicit analytic relations. Vectors $\boldsymbol{\omega}(t), \boldsymbol{\varepsilon}(t)$ and $\boldsymbol{\varepsilon}^{*}(t)$ are presented in analytic form at assigning splines $\varphi_{k}(t)$ by different degrees, in general case using three parts of given RM time interval $\mathrm{T}_{\mathrm{p}}^{r}$ : (i) initial part of the timeoptimized acceleration under constraints when the SC moves to its attitude motion with angular rate on fixed unit vector $\mathbf{e}_{3}$; (ii) the part for SC motion with a constant angular rate on the unit $\mathbf{e}_{3}$; (iii) the final part to guarantee the specified boundary conditions on the RM right end when the sixth order scalar splines $\varphi_{k}(t)$ are applied, moreover all parameters of these splines are computed by explicit analytic relations. As a result, for sequence of the SRs and RMs at the space imagery from current orbit, we obtain the uniform vector spline attitude guidance law which is a vector command signal for the spacecraft ACS.

In Fig. 11 we present the vector guidance law corresponding to the developed plan for areal land-survey of Istanbul neighborhoods, see Fig. 10. Here angles $\phi_{i}$ of the BRF orientation in the ORF, components of vectors $\boldsymbol{\sigma}(t), \boldsymbol{\omega}(t)$ and $\varepsilon(t)$ are marked by different colors - blue for roll, green for yaw and red color for pitch, and module of vector $\boldsymbol{\omega}(t)$ is marked by black color.

\section{SPACECRAFT ROBUST ATTITUDE CONTROL}

Assume that quaternion $\Lambda^{p}$, vectors of the angular rate $\boldsymbol{\omega}^{p}$ and acceleration $\varepsilon^{p}=\dot{\boldsymbol{\omega}}^{p}$ present the SC guidance law. Then the error quaternion is $\mathbf{E}=\left(e_{0}, \mathbf{e}\right)=\tilde{\boldsymbol{\Lambda}}^{p} \circ \boldsymbol{\Lambda}$, Euler parameters' vector is $\mathcal{E}=\left\{e_{0}, \mathbf{e}\right\}$, the error's matrix is $\mathbf{C}^{\mathrm{e}} \equiv \mathbf{C}(\mathcal{E})=\mathbf{I}_{3}-2[\mathbf{e} \times] \mathbf{Q}_{\mathrm{e}}^{\mathrm{t}}$ with matrix $\mathbf{Q}_{\mathrm{e}}=\mathbf{I}_{3} e_{0}+[\mathbf{e} \times]$, the error's vector is $\delta \phi=\left\{\delta \phi_{i}\right\}=$ $2 e_{0} \mathbf{e}$, and error $\delta \boldsymbol{\omega}=\left\{\delta \omega_{i}\right\}$ is defined as $\delta \boldsymbol{\omega}=\boldsymbol{\omega}-\mathbf{C}^{\mathbf{e}} \boldsymbol{\omega}^{p}$. Angular mismatch vector $\boldsymbol{\epsilon}_{l}=-\delta \phi_{l}, l \in \mathbb{N}_{0}$, is filtered with period $T_{p}$ and then the vector values $\epsilon_{k}^{\mathrm{f}}$ are applied in the developed digital control law for the GD cluster (Somov et al., 2005)

$$
\begin{gathered}
\mathbf{g}_{k+1}=\mathbf{B} \mathbf{g}_{k}+\mathbf{C} \boldsymbol{\epsilon}_{k}^{\mathrm{f}} ; \quad \tilde{\mathbf{m}}_{k}=\mathbf{K g}_{k}+\mathbf{P} \boldsymbol{\epsilon}_{k}^{\mathrm{f}} ; \\
\mathbf{M}_{k}^{\mathrm{g}}=\boldsymbol{\omega}_{k} \times \mathbf{G}_{k}^{\mathrm{o}}+\mathbf{J}\left(\mathbf{C}_{k}^{\mathrm{e}} \varepsilon_{k}^{p}+\left[\mathbf{C}_{k}^{\mathrm{e}} \boldsymbol{\omega}_{k}^{p} \times\right] \boldsymbol{\omega}_{k}+\tilde{\mathbf{m}}_{k}\right),
\end{gathered}
$$

where $\mathbf{C}_{k}^{\mathrm{e}}=\mathbf{C}\left(\mathcal{E}_{k}\right), \mathbf{G}_{k}^{o}=\mathbf{J} \boldsymbol{\omega}_{k}+\boldsymbol{H}_{k}$ and for $d_{u} \equiv 2 / T_{u}$, $a_{i} \equiv\left(d_{u} \tau_{1 i}-1\right) /\left(d_{u} \tau_{1 i}+1\right)$ elements of diagonal matrices $\mathbf{K}=\operatorname{diag}\left(k_{i}\right), \mathbf{B}, \mathbf{C}$ and $\mathbf{P}$ are computed by the relations $b_{i} \equiv$ $\left(d_{u} \tau_{2 i}-1\right) /\left(d_{u} \tau_{2 i}+1\right) ; p_{i} \equiv\left(1-b_{i}\right) /\left(1-a_{i}\right) ; c_{i} \equiv p_{i}\left(b_{i}-a_{i}\right)$ with adaptive-robust tuning the parameters $\tau_{1 i}, \tau_{2 i}$ and $k_{i}$. The GMC control torque vector $\mathbf{M}_{k}^{\mathrm{g}}(10)$ is "re-calculated" into vector $\mathbf{u}_{k}^{g}$ of the GD commands using explicit function of the AM distribution between four gyrodines (Somov et al., 2005). These commands are fixed at the current step of digital control with period $T_{u}$. The GMC is unloaded from accumulated AM by the compensation scheme with digital control of a magnetic actuator.

\section{SIMULATION OF THE ACS OPERATION}

Applied astroinertial ADS was simulated with the following periods: $T_{q}=(1 / 128) \mathrm{s}, T_{o}=1 \mathrm{~s}$ and $T_{p}=(1 / 8) \mathrm{s}$. The discrete 


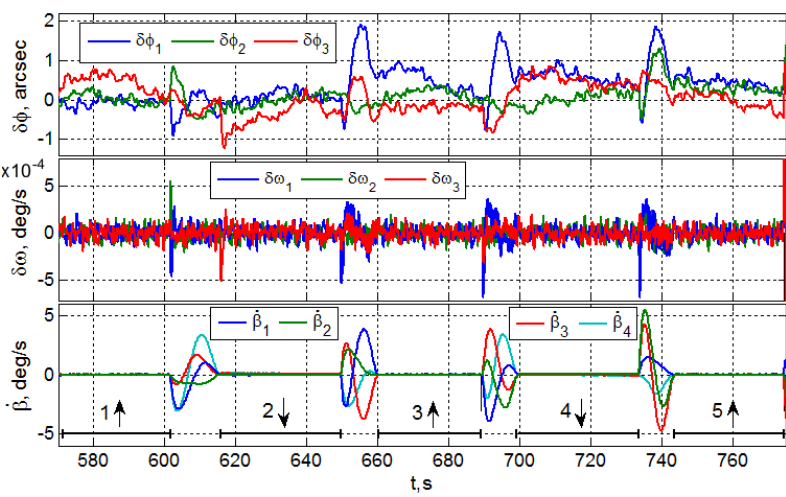

Figure 12. Errors at areal land-survey and the GD angular rates

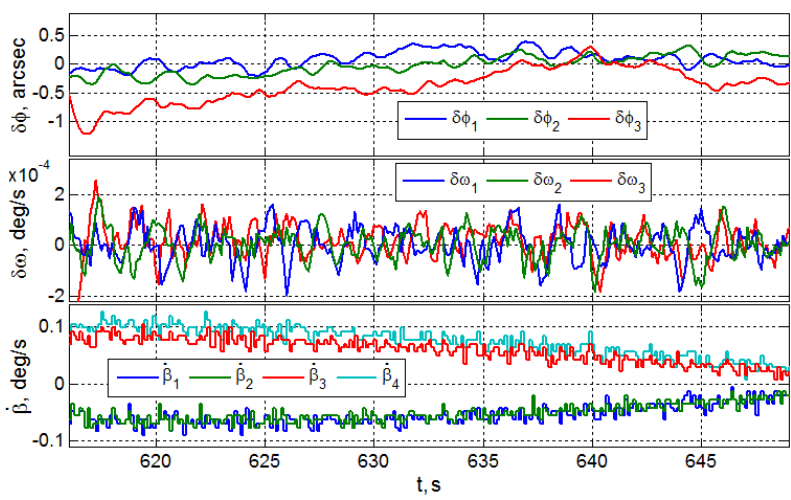

Figure 13. Errors during second OSR and the GD angular rates

filtering of vector $\boldsymbol{\epsilon}_{l}$ was executed with period $T_{p}=(1 / 8) \mathrm{s}$, and the GMC digital control was formed with period $T_{u}=(1 / 4) \mathrm{s}$. In Figs. 12 and 13 we present errors at stabilization of the SC angular motion during the areal land-survey (Fig. 10) with the uniform attitude guidance law presented in Fig. 11, and also the GD angular rates. In the lower part of Fig. 12 we have pointed the time intervals of the OSMs with their indexes and scanning directions.

\section{CONCLUSIONS}

We briefly have presented new results on guidance, precise attitude determination and robust gyromoment attitude control of an agile land-survey satellite during operative optoelectronic areal imagery for disaster management. We represented the discrete algorithms developed for onboard signal processing, alignment of the telescope with the star tracker cluster, and also onboard algorithms for alignment and calibration of the spacecraft attitude determination system. We have developed methods for a planning the areal land-survey and synthesis of SC vector spline guidance laws both at a spacecraft optoelectronic observing routes and its spatial rotational maneuvers with given boundary conditions. We briefly have presented algoritms for dicrete filtering and robust digital attitude control of an agile land-survey satellite, and also numerical results on the efficiency of the developed algorithms for a satellite attitude control system. These results were obtained for the areal land-surveying of Istanbul neighborhoods by a sattelite in sun-synchronous orbit with altitude of $720 \mathrm{~km}$. The areal land-survey with terrestrial dimension $200 \times 203 \mathrm{~km}$ was performed using five orthodromic partly overlapping scanning routes with rotational maneuvers in-between and total duration of 225 seconds only. Within 20 minutes the obtained video data together with a service information on the actual conditions of the areal imagery will received and processed at terrestrial space centre. As a result, the government agencies will have current information on the actual influence of the natural disaster.

According to statistics, in Istanbul the earthquake occurs every 50 years, and every 300 years they literally wipe the city off the face of the Earth. The reason is that Istanbul is located in the zone of the North Anatolian fault, one of the largest and most active in the world. In the last 2000 years in the region occurred more than 30 earthquakes with magnitude more than 7 points. In 1999, the earthquake whose epicenter was $11 \mathrm{~km}$ from Izmit and $80 \mathrm{~km}$ from Istanbul, caused the death of 19,000 people and damage to many historical monuments, museums, and libraries. These tremors were felt both in Turkey and in Russia.

Today there is a very high risk of earthquakes in the Marmara region where $50 \%$ of the production means are located and lives a quarter of the Turkey population. Seismologists have conducted a study using the GPS marks located on those places where an earthquake was registered in the Marmara sea, and also underwater equipment. The measurements will allow to constantly monitor the seismic activity and to improve the early detection and rapid response. Although these measures help to better study and understand the nature of earthquakes, but this system will raise the alarm just 12 seconds before the tremors start. Therefore, the use of the space observation technology is very important for quick actions during natural disasters including earthquakes.

\section{ACKNOWLEDGMENTS}

This work was supported by grants of Russian Foundation for Basic Research (14-08-91373, 17-48-630637) and the Scientific and Technological Research Council of Turkey (113E595).

\section{REFERENCES}

Lanczos, C., 1956. Applied Analysis. Englewood Cliffs.

Markley, F. L. and Mortar, D., 2000. Quaternion attitude estimation using vector observation. The Journal of the Astronautical Sciences 48(2\&3), pp. 359-379.

Orfanidis, S., 1996. Introduction to Signal Processing. Englewood Cliffs, Prentice-Hall.

Shampine, L., 1986. Some practical Runge-Kutta formulas. Mathematics of Computation 46(173), pp. 135-150.

Somov, Y. and Butyrin, S., 2012. In-flight alignment of a space telescope and a star tracker cluster at a scanning observation of the Earth marked objects. In: Proceedings of 19th Saint Petersburg International Conference on Integrated Navigation Systems, Saint Petersburg, pp. 242-244.

Somov, Y., Butyrin, S. and Skirmunt, V., 2008. In-flight alignment calibration of a space telescope and a star tracker cluster. In: Proceedings of 15th Saint Petersburg International Conference on Integrated Navigation Systems, Saint Petersburg, pp. 139-143.

Somov, Y., Butyrin, S., Somov, S. and Hajiyev, C., 2017. Precise astroinertial attitude determination of a maneuvering land-survey satellite. In: Proceedings of 8th International Conference on Recent Advances in Space Technologies, Istanbul, pp. 409-413.

Somov, Y., Platonov, V. and Sorokin, A., 2005. Steering the control moment gyroscope clusters onboard high-agile spacecraft. In: Automatic Control in Aerospace, Vol. 1, Elsevier Ltd., Oxford, pp. 137-142. 